\title{
Case Report 220
}

Dorit D. Adler, M.D., Caroline E. Blane, M.D., and John F. Holt, M.D.

Division of Pediatric Radiology, C.S. Mott Children's Hospital, University of Michigan Medical Center, Ann Arbor, Michigan, USA
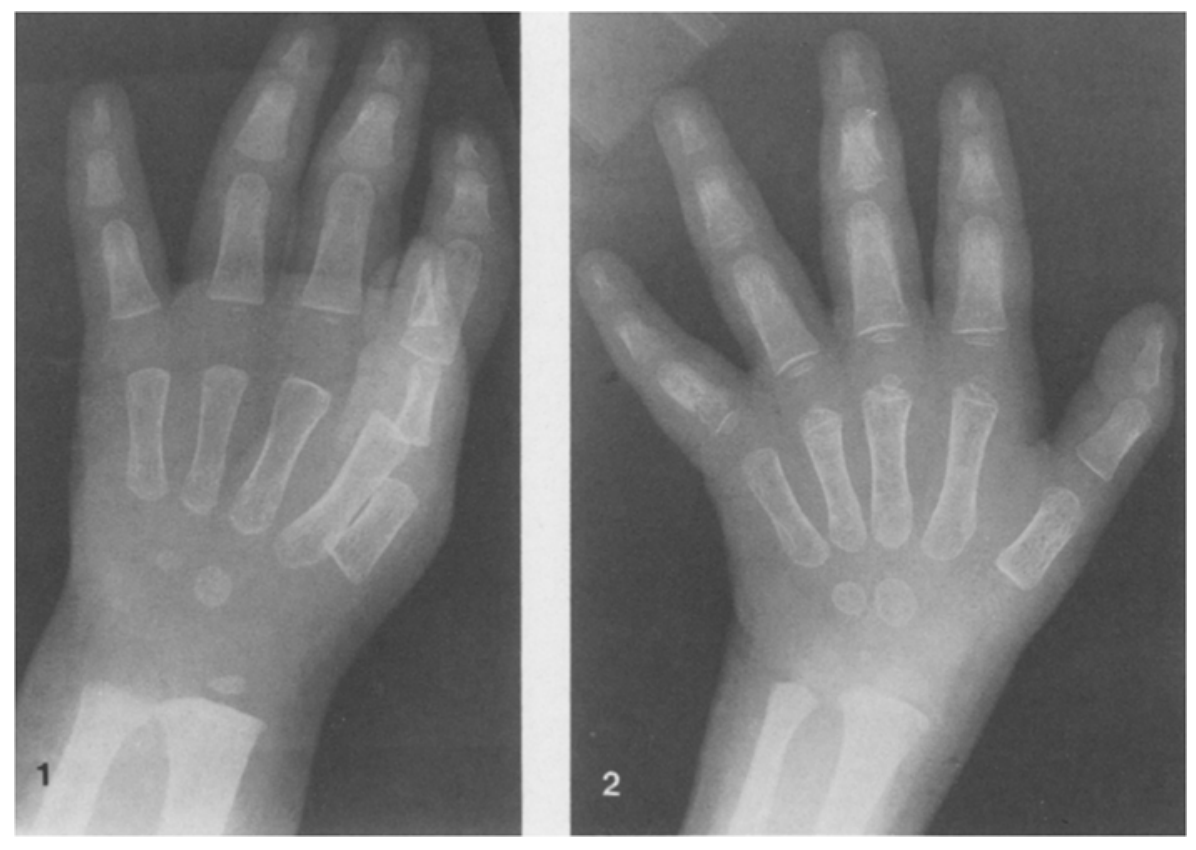

\section{Radiological \\ Studies}

Fig. 1. A posteroanterior roentgenogram of the left hand at the age of 13 months shows no abnormality

Fig. 2. A repeat PA film of the left hand at the age of 27 months shows a permeative, destructive process of the proximal phalanx of the fifth finger, mainly involving the proximal half of the diaphysis, metaphysis and very likely even the ossification center. No periosteal reaction or joint involvement is identified

\section{History}

This 13-month-old male child of Northern European extraction was admitted to hospital with a three month history of fever and a skin rash. Nonpuritic erythematous, papular lesions had been noted on the anterior surfaces of both thighs, extending over the trunk and extremities. Subcutaneous nodules had developed beneath the original lesions. Laboratory investigation was normal including ANA, $\mathrm{CH}-50$ and angiotensin-coverting enzyme. All bacteriological cultures were negative. Among other areas examined it is expecially important to note that radiological study of the left hand at the time

Address reprint requests to: C.E. Blane, M.D., Division of Pediatric Radiology, C.S. Mott Children's Hospital, University of Michigan Medical Center, Ann Arbor, MI 48109, USA of this first admission was considered normal (Fig. 1). The chest was normal radiologically.

A biopsy of one of the skin lesions was obtained.

At the age of 18 months the child returned with multiple, yellowish papules on the extremities. A repeat skin biopsy was performed.

The child was observed again at the age of 27 months. He was febrile, with erythema virtually covering the entire body. The child complained of joint pain at various sites. On examination soft tissue thickening of both wrists with swelling of the left fifth finger was noted. Roentgenograms at this time showed a reticulated, destructive pattern involving the proximal phalanx of the left fifth finger without joint involvement or evidence of periosteal reaction (Fig. 2). 


\section{Diagnosis: Osseous Sarcoidosis of Left 5th Digit in a Young Child with Systemic Sarcoidosis}

The differential diagnosis includes pyogenic osteomyelitis, tuberculous dactylitis, fungal diseases (e.g., cryptococcosis, blastomycosis, histoplasmosis, coccidioidomycosis) and eosinophilic granuloma. Much less likely possibilities include Ewing tumor and metastatic disease (neuroblastoma).

\section{Discussion}

The skin biopsy specimen showed a noncaseating granuloma with multinucleated giant cells (Fig. $3 \mathrm{~A}$ and $B$ ), consistent with sarcoidosis.

The osseous lesions of sarcoidosis are uncommon in children. Radiological evidence of skeletal involvement under the age of four years is particularly unusual. However uncommon the lessions of the skeletal system may be in children, when they are observed they are of significant diagnostic value. The classical appearance of a reticulated, permeative pattern of destruction of tubular bones of the hands or feet without joint involvement and virtually always without periosteal reaction must suggest sarcoidosis. The infant reported here with the radiological pattern of the destructive process in the left fifth finger observed at the age of 27 months is one of the youngest individuals with sarcoidosis in the literature and the first to be reported at this age in more than two decades.

Holt and Owens reported radiological evidence of osseous sarcoid in $16 \%$ of their patients in 1949 [1]. The largest and most current review of adult sarcoidosis reported $3 \%$ of patients with sarcoidosis to have skeletal involvement [2]. A major review with a report of 113 children with sarcoidosis by McGovern and Merritt reported skeletal lesions in 33 of the 113 children or $29 \%$ [3]. The range in ages of these children with bone lesions was 11 months to 15 years. Only four cases have been reported of skeletal involvement with sarcoidosis under the age of four years [3].

Skeletal involvement is rare in the absence of skin lesions. Patients with osseous sarcoid usually have chronic fibrotic sarcoid at other sites [2]. The patients with osseous sarcoid have a four fold increase in mortality, which probably reflects the multisystem involvement [2].

The osseous lesions in sarcoidosis are divided into three major radiological patterns: (1) a stippled pattern with diffuse enlargement of the bony lacunae and subsequent rarefaction or actual destruction; (2) progression of the rarefaction or destruction, particularly in the distal ends of the distal phalanges resulting also in the development of "punched-out" cyst-like lesions; and (3) the most common and even specific radiological appearance which is a reticular, lattice-work pattern of bone destruction [1]. Holt and Owens pointed out two important features of bone lesions due to sarcoidosis: (1) the relative lack of periosteal reaction, and (2) the lack of radiological evidence of joint involvement, usually [1].

In this child no radiological evidence of involvement of the chest was noted. This brings up the issue of the reluctance to diagnose sarcoidosis of the skeletal without chest involvement. In this connection, the series by McGovern and Merritt, reporting 33 cases of skeletal sarcoid in childhood, showed 8 children without involvement of the chest. In the article by Holt and Owens one of the cases of osscous sarcoid did not have involvement in the chest. In a case report and review of the literature by Brodey et al. [3] a single case in an adult of skeletal sarcoid without involvement of the chest was reported. A large series of 81 adults with osseous sarcoidosis by Israrel and Sones showed no evidence of chest involvement in 12 patients.

In summary, the typical manifestation of osseous sarcoid radiologically in this two year old child reinforces the clinical and pathological diagnosis of sarcoidosis. This confirmatory evidence was considered of great importance in this child, harboring a disease which is rare in this age group.

\section{Editorial Comment}

It must be acknowledged that although the histological apearance in this case is considered diagnostic of sarcoidosis, the finding of noncaseating granuloma with multinucleated giant cells is probably nonspecific and may be observed in a variety of disorders (e.g. end stage histiocytosis). In the context of the other features in this child it is considered fully justifiable to consider the case presented as sarcoidosis. 


\section{Pathological Studies}
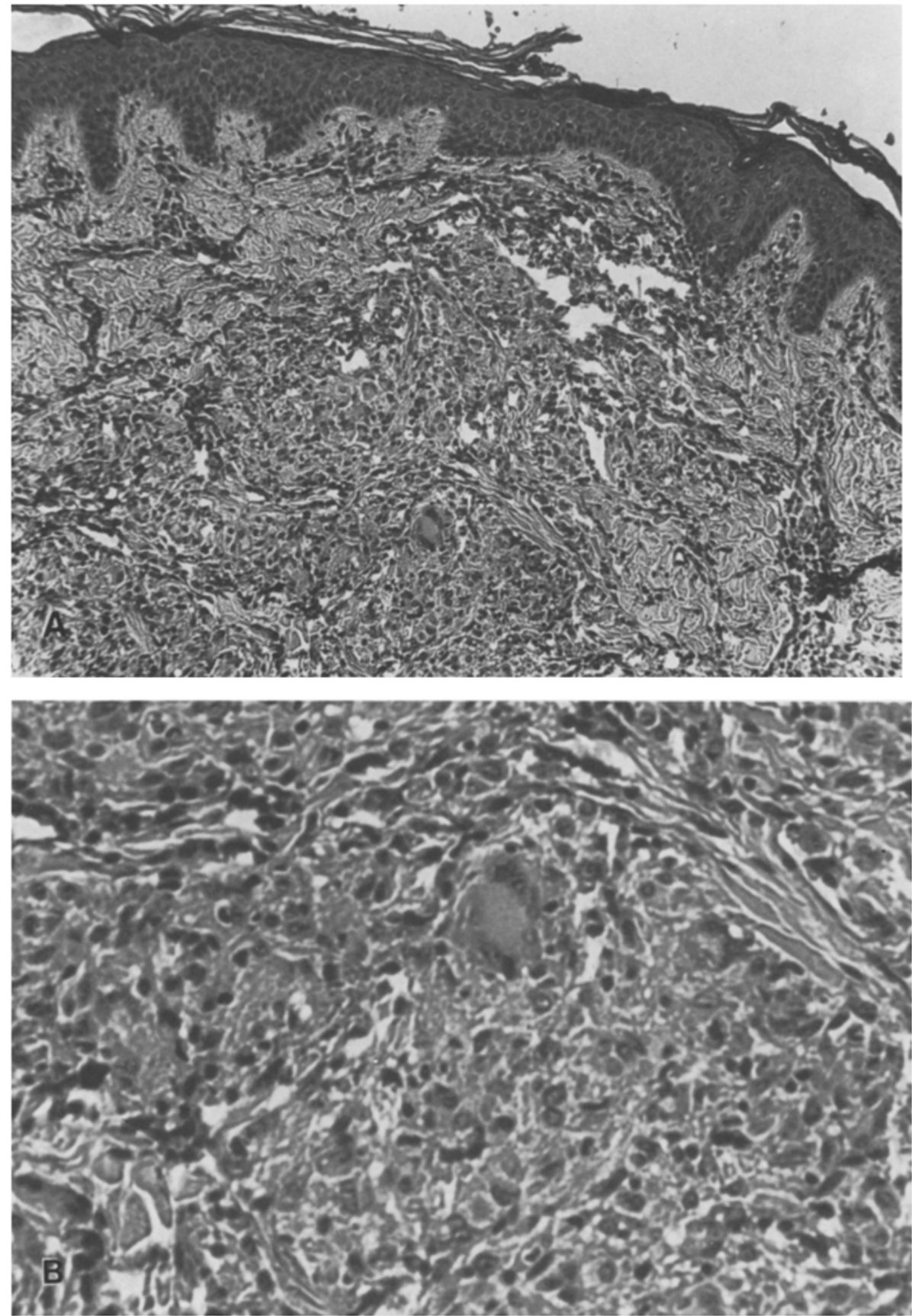

Fig. 3A, B. Photomicrographs of the specimen obtained from the skin biopsy (H \& E stain - low and high power) show the typical sigmata of noncaseating granuloma with multinucleated giant cells

\section{References}

1. McGovern JP, Merritt DH (1956) Sarcoidosis in childhood. Adv Pediatr 8:97

2. Holt JF, Owens WI (1949) The osseous lesions of sarcoidosis. Radiology 53:11 (1949)

3. Brodey PA, Pripstein S, Strange G, Kohout ND (1976) Ver- tebral sarcoidosis. A case report and review of the literature. AJR 126:900

4. Israel HL, Sones M (1958) Sarcoidosis. Clinical observation on one hundred sixty cases. Arch Intern Med 102:766 